\title{
The influence of audio communications
}

\section{technology on computer-supported collaborative learning}

\author{
Denise Whitelock, Eileen Scanlon and Josie Taylor \\ Institute of Educational Technology, The Open University
}

Our study set out to investigate how collaborative learning between pairs of adult subjects solving physics problems with a computer is effected when the subjects are not co-present but have an audio and computer link only. It also examined how the groups' gender composition could influence both group productivity and individual learning. This paper reports that all pairs performed significantly better in the audio condition with respect to on-task problem-solving measures, and pre-to post-test change scores.

\section{Introduction}

In recent years there has been an appreciation of the benefits that can be obtained by students working in groups or teams using computers (Eraut and Hoyles, 1988). But there is a difference in opinion as to how a partner enhances learning, and in particular how well adult learners do in the collaborative setting. In order to capitalize on the opportunities offered by new technologies, we need to understand more fully how the process of collaboration is effected by different communication technologies, and how the technologies themselves might be used to best advantage for the benefit of distance learners. However, another factor, apart from the technology itself, which is thought to influence computer-supported collaborative learning is in the gender distribution of the group. A number of classroom studies have shown gender differences when children work together with computers, and these have been reported from a number of subject-domains including science (Scanlon et al, 1993; Littleton et al, 1992). Since our own expertise is in the area of science learning, we selected a non-trivial physics task for the subjects to work with, and that is in the area of elastic collisions. Previous studies (Villani and Pacca, 1990; Whitelock et al, 1993) have shown that both adults and school-children have difficulty in predicting the subsequent motion of balls or ice pucks after they collide. Villani's study stresses that even postgraduate students tend to revert to their informal commonsense notions unless they are cued to use formal representations of these types of problem. These studies have demonstrated that the topic of elastic collisions is a complex yet fruitful one in which to engage students in group work. 
The piece of research reported in this paper set out to investigate two important questions with respect to communication technologies and adult learners. These were:

1. Do pairs of students perform better when they work side by side, with a physics simulation, than when their only contact with one another is via a telephone and their respective computers?

2. Does group composition with respect to gender affect task performance in the above two conditions?

With these questions in mind, our objectives included monitoring both group productivity and individual learning in both the audio and side-by-side conditions, nd investigating whether the influence of gender had any identifiable consequences on both these outcomes.

\section{Method}

\section{The computer simulation}

The PuckLand simulation version 2.6 was written in Hypercard for use with the Apple Macintosh. It uses a direct manipulation approach which allows students to investigate a series of collisions between two ice pucks. It consists of a pair of pinball-style flippers on either side of the screen with which subjects can flick pucks. The amount of force with which the flippers hit the pucks can be varied, as can the mass of the pucks. The pucks, ranging from 1 to 100 units of mass, can be dragged into position ready to be struck by the flippers.

The initial velocity of the collision is controlled by directly manipulating the angle of the flipper from 90 to 180 degrees in the vertical plane. Before the Go button could be activated, subjects have to qualitatively predict the speed and direction of the pucks after collision. When the Go button is pressed, the pucks move towards each other on the screen, and are animated with a speed proportional to that set by the initial angle of impact executed by the flipper. The program also highlights the icons with the correct answers after the experiment is run.

After the pucks collide, they move away from each other with a speed which is calculated from the correct physics formalisms. This means that the principles of conservation of momentum and kinetic energy are obeyed, and again the apparent screen velocities of the pucks is proportional to their calculated values. At the bottom of the screen is a grid which provides numerical information about the amount of energy and momentum that the system had initially, and then, subsequent to being run, it shows what the effect of the collision is on these two factors. Every experiment attempted by users of the simulation is automatically logged by the computer.

The networked version of PuckLand has a communication protocol written in Hypertalk 2 which allows two to eight machines to be simultaneously linked together. With this system you cannot continuously see your partner's screen or take control of it, as with other communication protocols such as Timbuktu. Although screens can be exchanged between the partners, this was not an automatic process, since all subjects could choose to accept or reject data from their partner. This latter feature was incorporated into the system to prevent one partner, who might have more computer or physics experience than the other, dominating the proceedings. 


\section{Using the simulation}

All subjects had access to their pre-test predictions about the motion of the ice pucks in a number of given scenarios, during the experimental period. They were explicitly requested to work together as a team while using the simulation, in order to aid their joint understanding of elastic collisions. They were given time at the beginning of the session to explore the simulation and to try out any scenarios that interested them. Once the subjects felt they understood the domain and had exploited the simulation as an information resource, they were asked to solve three different problems. These problems required the subjects not to predict the result of a collision but to state the original conditions for a given outcome. The problems were posed in order of difficulty and were as follows:

1. What initial conditions are needed to send the pucks travelling away from each other at the same speed?

2. What initial conditions are needed to stop one puck after impact?

3. What initial conditions are needed to make pucks of unequal masses move away, after impact, at the same speed?

All subjects were video-recorded throughout the experimental sessions, and the subjects' dialogue in the audio condition was tape-recorded on a telephone answering machine. The experiments which were run with the simulation were logged, and the networked version of PuckLand also recorded subjects requests to exchange screens. The program finally logged the screens which were accepted or rejected by each partner.

\section{Subjects}

All subjects in this study were adults. They held a first degree or equivalent professional qualification, and $89 \%$ of them had experienced some form of physics education. The mean age of the group was 39.9 years $(s d=11.8)$.

The subjects were paired with people they knew and were happy to collaborate with. They formed both single-sex and mixed-gender dyads. They were assigned to the side-by-side or audio condition depending on which of two test sites they could more easily reach. The numbers in each group are shown in Table 1. However, some of the pairs had worked together before while others had not. The main factor in determining this relationship was whether subjects were available to participate in the experiment on the same day. We aimed for a minimum of 12 subjects in each condition, which was achieved comfortably, but the final number was determined by the availability of subjects during the experimental period.

\section{Procedure}

The subjects were pre-tested with a version of a questionnaire developed by Whitelock $e t$ al (1993). This included a prediction task where subjects were asked to predict the subsequent motion of two ice pucks after collision for the following five conditions:

Condition 1: Puck A is small, light and moving; Puck B is larger, heavier and stationary.

Condition 2: Puck A is large, heavy and moving; Puck B is small, light and stationary.

Condition 3: Puck A and Puck B are identical in size but only Puck A is moving. 


\begin{tabular}{|lll|}
\hline & Subject group & $\mathbf{n}$ \\
& & \\
Side by side & Male & 14 \\
Side by side & Female & 16 \\
Side by side & Mixed & 18 \\
& & \\
Audio & Male & 12 \\
Audio & Female & 12 \\
Audio & Mixed & 16 \\
\hline
\end{tabular}

Table 1: Numbers of subjects who worked in the audio-only or side-by-side condition when using the physics simulation

Condition 4: Puck A is small, light and moving; Puck B is larger, heavier and moving.

Condition 5: Puck A and Puck B are identical in size and both are moving.

The subjects were not only asked about their understanding of kinetic energy and momentum, but also about their expectations of the computer task. In the post-test, all the questions were repeated and some extra information was obtained about the subjects' perceptions of the whole experience.

\section{Results}

The key finding from this experiment is that all the pairs did significantly better in the audio rather than in the side-by-side condition (see Figure 1). A two-way ANOVA performed on the pre-post test change scores revealed a significant difference for the communication condition $(\mathrm{F}=11, \mathrm{p}<0.001$ ), but there was no overall difference according to gender.

As well as simply looking at improvement on pre- to post-test scores, it was possible to investigate problem-solving success while subjects were using the simulation (see Figure 2). The three problems were given in order of difficulty and all the pairs were able to solve Problem 1. Problem 2 proved to be difficult only for the mixed-gender group in the side-byside condition, while a solution to Problem 3 was more frequently obtained by all groups working in the audio condition. A two-way ANOVA performed on the problem-solving scores revealed a significant difference for the communication condition $(F=15, p<0.001)$, but there was no overall difference according to gender. There was no significant difference in the amount of time spent working with the simulation for all the groups tested. Therefore further analysis of the number of experiments performed was undertaken to find reasons for these group differences.

As for the number of experiments performed in each communication condition for each gender group studied (see Figure 3), the male pairs conducted more experiments in the side-by-side than in the audio condition. The reverse result is true for the female pairs, while subjects of mixed gender carried out exactly the same number of experiments regardless of the communication channel. A two-way ANOVA performed on the number of experiments performed revealed no overall difference according to communication or gender condition.

With respect to subjects' management and exchange of experimental information in the audio 


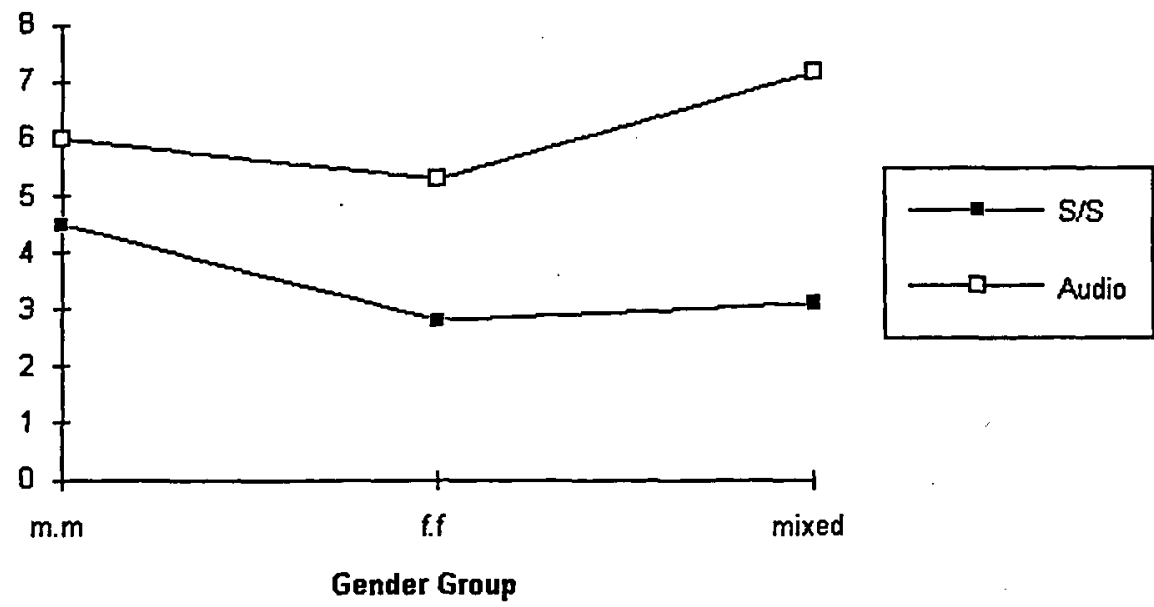

Key: $m \cdot m=$ male; $f f=$ female; mixed $=$ mixed gender

Figure l: Groph to illustrote change scores versus mode of communication and gender mix

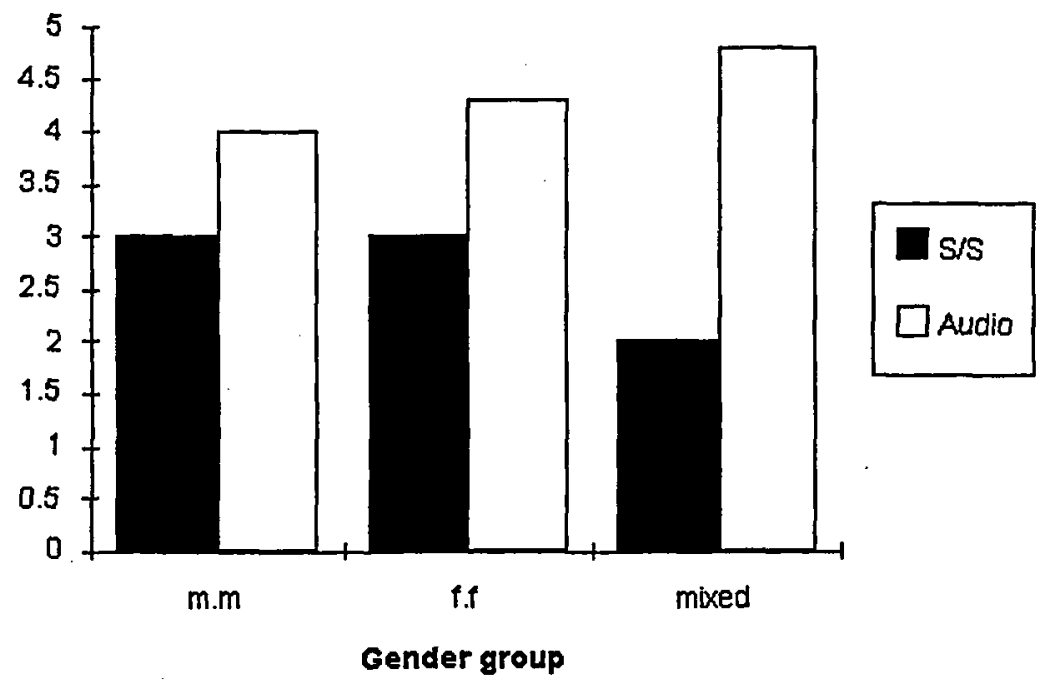

Key: $m \cdot m=$ male; $f f=$ female; mixed $=$ mixed gender

Figure 2: Graph to illustrate problem-solving scores versus mode of communication and gender mix

condition, only information on $25 \%$ of the experiments attempted was transmitted to partners during the session (see Figure 4). From this small number, only four screen-updates were rejected. This suggests that the exchange-screen facility was used only when subjects assumed it would be mutually beneficial. The videos were consulted to ascertain what sorts of scenario prompted partners to send screen-data to one another. Two types of events prompted this 


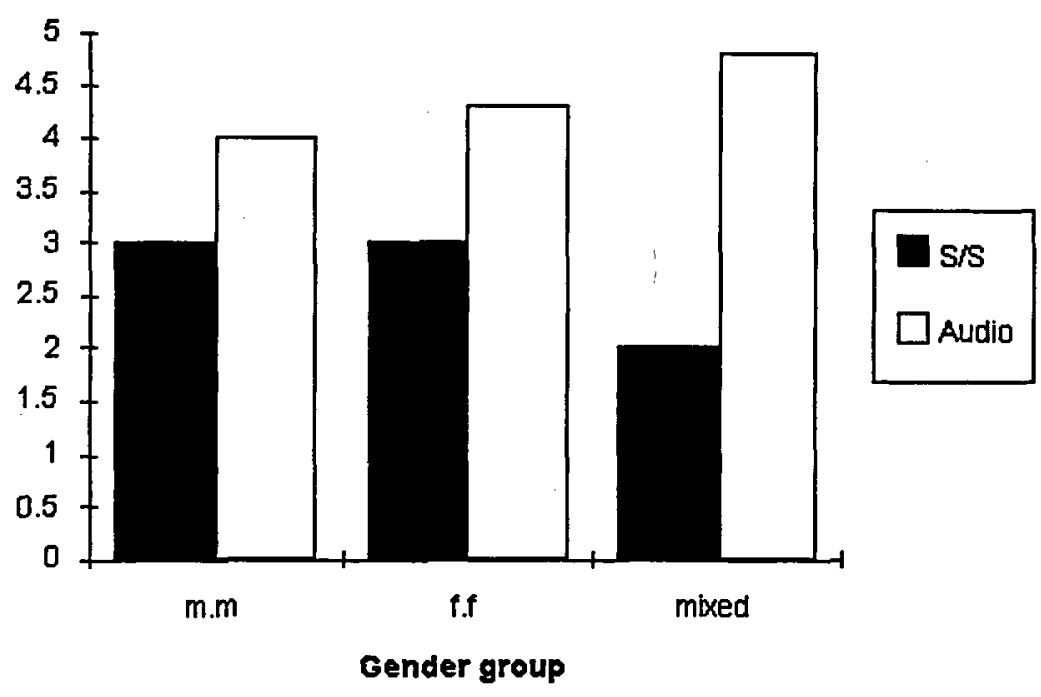

Key: $m \cdot m=$ male; $f f=$ female; mixed $=$ mixed gender

Figure 3: Graph to illustrate mean number of experiments versus mode of communication and gender mix

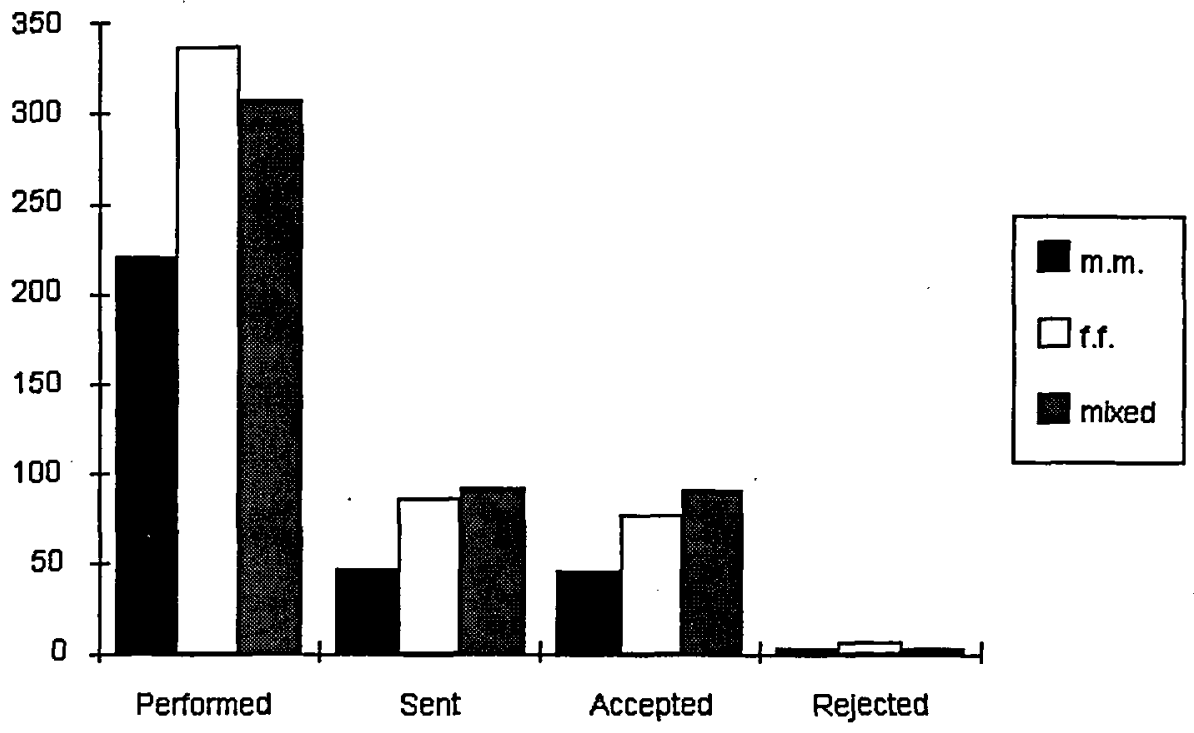

Experimental interactions between the subjects

Key: $m \cdot m=$ male; $f f=$ female; mixed $=$ mixed gender

Figure 4: Graph to illustrate number of experiments exchanged when subjects were working in the audio condition only 
course of action, and these occurred when a partner wanted to help a floundering colleague or when one of the subjects had a particularly interesting finding to communicate.

The main difference between subjects' behaviour during the audio session on the one hand, and in the side by side condition on the other is that the 'audio' pairs did not choose to continually work through everything together. They branched off on their own at times, and explored their individual hunches or hypotheses about what they thought was going on. They then came back together again to discuss their findings. In fact, this was a strategy employed by the most successful problem-solvers. An extract from the transcript of one of the male/male pairs illustrates this particular modus operandi:

1. One way of using the resources, as it were, would be if we both played around independently without communicating back too much about it.

\section{And then come back.}

1. Come back and compare notes. All right?

\section{All right.}

1. So we're still trying to create a situation where one of the pucks is stationary after impact; all right, are you happy to do that?

\section{Yes I am.}

1. Right, let's pursue a little hunt here and then maintain radio silence.

This strategy certainly contributed to subjects in the audio condition solving more problems while working together online than subjects in the face-to-face condition (see Figure 2). However, some of the problems were solved not only by students testing hypotheses, but by also adopting a trial-and-error strategy. This latter course of action would also increase the number of experiments performed with the simulation.

\section{Conclusions}

The results indicate that with this particular computer-based investigation subjects working in the audio condition were more likely to improve their post-test scores and to succeed in the problem-solving exercises than subjects working side by side. We have found that it is not just a difference in the number of experiments but in the type of experiments attempted that contributed to joint problem-solving success. These results are also a reflection of how the subjects chose to work together in the different communication conditions. For example, in the audio condition, because each subject had his or her own computer, it was possible for individuals to explore their own different hypotheses when their joint deliberations proved unsuccessful. We are in the process of analysing the discourse of the pairs of subjects throughout the experimental sessions, and a preliminary pass at the data suggests that cooperative construction of shared meaning (Barbieri and Light, 1992) rather than conflict is more important for successful collaboration.

This study did not reveal any significant difference in group problem-solving results on individual pre- to post-test change scores with respect to gender, but there was more improvement in the mixed-gender pairs change scores in the audio than in the side-by-side 
condition. One possible explanation for this is that there was more male dominance when couples were working side by side. However, in the audio condition, both males and females were testing out their own predictions. Our findings suggest gender does not influence learning with this particular computer task but that subjects in certain communication settings benefit more than others. And rather than being an inferior substitute to working side by side, it seems that the audio condition had some advantages.

This study forms part of an ongoing research programme, and we will investigate students solving the same physics problems, working at a distance, but this time linked by a video conferencing system.

\section{References}

Barbieri, S. and Light P. (1992), 'Interaction, gender and performance on a computer-based problem-solving task', Learning and Instruction, 12, 199-213.

Eraut, M. and Hoyles, C. (1988), 'Group work with computers', Journal of Computer Assisted Learning, 5, 12-24.

Littleton, K., Light, P., Joiner R., Messer, D. and Barnes P. (1992), 'Pairing and gender effects in computer-based learning', European Journal of Psychology of Education, 7 (4), 1-14.

Scanlon, E., O'Shea, T., Hennessy, S., Taylor, J. and Whitelock, D. (1993), 'Gender, computers and group work in science', 7th Conference on Gender, Science and Technology, GASAT, August 1993.

Whitelock, D., Taylor, J., O'Shea, T., Scanlon, E., Clark, P. and O'Malley, C. (1993), 'Challenging models of elastic collisions with a computer simulation', Journal of Computers in Education, 20 (1), 1-9.

Villani, A. and Pacca, J.L.A. (1990), 'Spontaneous reasoning of graduate students', International Journal of Science Education, 12, 589-600. 